\section{Acute pancreatitis after endoscopic biopsy of the minor duodenal papilla in an individual with pancreas divisum}
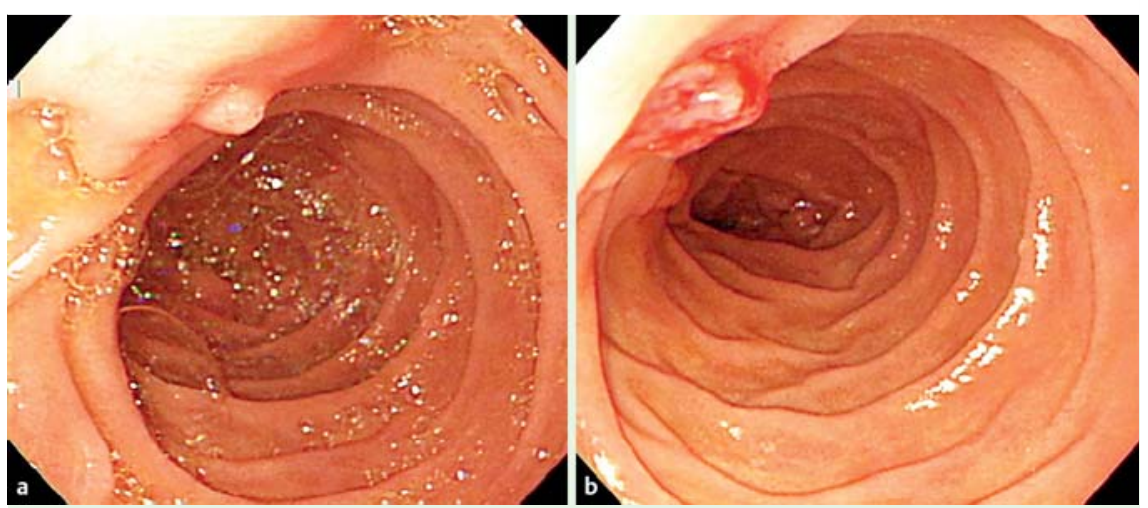

Fig. 1 a Esophagogastroduodenoscopy in a healthy 47-year-old man showed a 4-mm "polyp" in the second portion of duodenum, proximal to the major duodenal papilla. $\mathbf{b}$ Appearance after endoscopic biopsy.

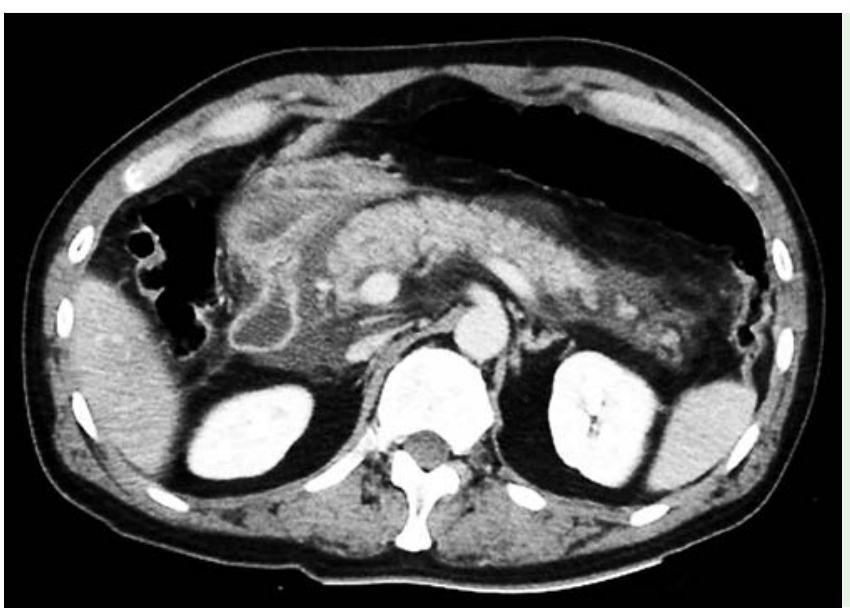

Fig. 2 Abdominal computed tomography (CT) scan showed diffuse pancreatic swelling with a peripancreatic fluid collection.

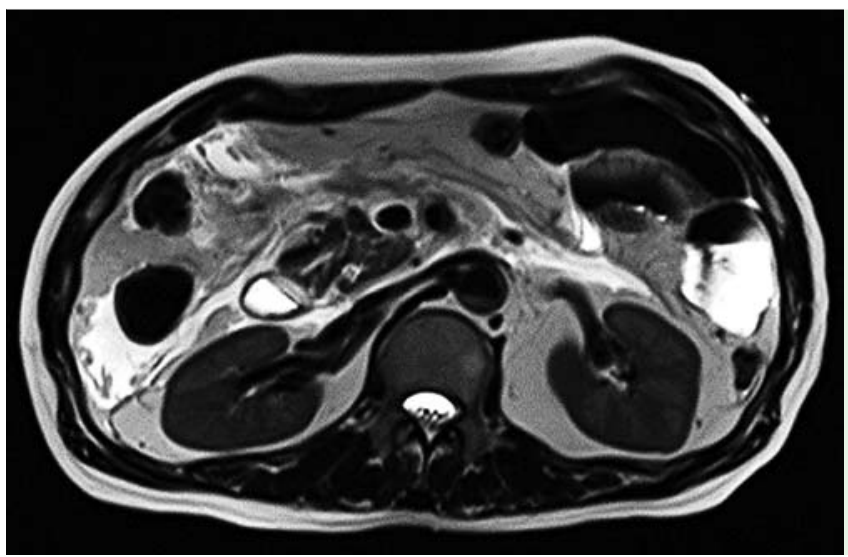

A healthy 47-year-old man underwent esophagogastroduodenoscopy (EGD) for general examination. A small polyp 4 $\mathrm{mm}$ in size was seen in the second portion of the duodenum; its location was proximal to the major duodenal papilla ( $\bullet$ Fig.1a). An endoscopic biopsy was taken ( $\bullet$ Fig.1b). The biopsy findings showed normal duodenal mucosa.

The patient developed severe epigastric pain 3 hours after the EGD. Serum amylase and lipase levels were markedly increased (8860U/L and 2640U/L, respectively), while other serum biochemical levels were within normal ranges. Abdominal computed tomography (CT) scan showed diffuse pancreatic swelling with a peripancreatic fluid collection ( $\bullet$ Fig.2). Magnetic resonance imaging of the pancreas and magnetic resonance cholangiopancreatography showed that the common bile duct and the dorsal pancreatic duct had different duodenal openings and that the dorsal pancreatic duct drained into the minor duodenal papilla with no communication with the common bile duct ( Fig.3, $\bullet$ Fig.4). We therefore diagnosed pancreas divisum. The patient's abdominal pain was improved by intravenous fluid hydration and restriction of food. He was discharged 15 days later.

On the basis of many autopsy studies, pancreas divisum is found in $8 \%$ of the normal population [1]. In cases of pancreas divisum, the small ventral duct drains via the major papilla and the large dorsal duct drains via the minor papilla. Most of the pancreatic ductal system drains via the minor papilla. Therefore papillary edema due to endoscopic biopsy may obstruct the pancreatic duct causing acute pancreatitis. Ductal obstruction can easily develop as a result of relative stenosis of the minor papilla in cases of pancreas divisum [2,3]. In the case reported here, the opening of the minor papilla distinctly resembled a polyp. If acute pancreatitis develops after endoscopic biopsy of the minor papilla, the possibility of pancreas divisum should be checked.

Endoscopy_UCTN_Code_CPL_1AK_2AB

Competing interests: None

\section{Woo Jin Jeong}

Division of Gastroenterology, Department of Internal Medicine, Gangneung Asan Hospital, University of Ulsan College of Medicine, Gangneung, Korea 

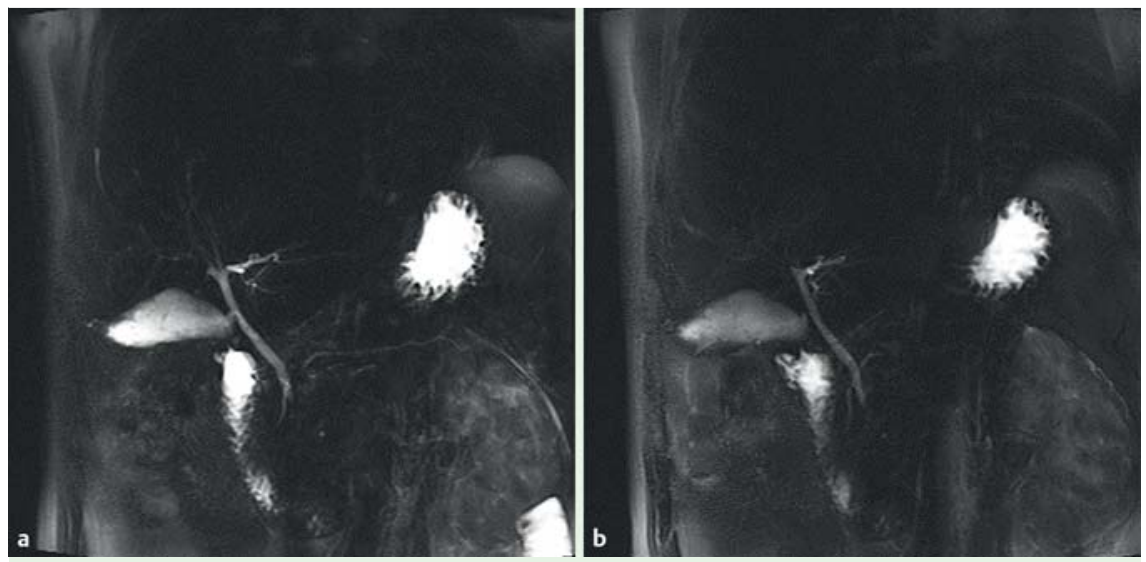

Fig. 4 Coronal magnetic resonance cholangiopancreatography showed the dorsal pancreatic duct superior to the common bile duct and draining independently through the minor papilla, with no communication between the ductal systems.

\section{References}

1 Fogel EL, Toth TG, Lehman GA et al. Does endoscopic therapy favorably affect the outcome of patients who have recurrent acute pancreatitis and pancreas divisum? Pancreas $2007 ; 34: 21-45$

2 Gincul R, Ciocirlan M, Dumortier J et al. Severe acute pancreatitis following endoscopic biopsy of the minor duodenal papilla. Endoscopy 2009; 41: E195 -E196

3 Jeong WS, Kim HJ, Shim CN et al. A case of acute pancreatitis after endoscopic biopsy in congenital pancreatic duct anomalies. Korean J Med 2012; 82: 709-712

\section{Bibliography}

DOI http://dx.doi.org/

10.1055/s-0042-109699

Endoscopy 2016; 48: E238-E239

(c) Georg Thieme Verlag KG

Stuttgart · New York

ISSN 0013-726X

\section{Corresponding author}

\section{Woo Jin Jeong, MD}

Division of Gastroenterology,

Gangneung Asan Hospital

38, Bangdong-gil, Sacheon-myeon

Gangneung-si, Gangwon-do

Korea, 210-711

Fax: +82-33-6104960

jwoojini73@gmail.com 